\title{
Canonical transformations to action and phase-angle variables and phase operators
}

\author{
A. Luis and L. L. Sánchez-Soto \\ Departamento de Optica, Facultad de Ciencias Físicas, Universidad Complutense, 28040 Madrid, Spain
}

(Received 12 January 1993)

\begin{abstract}
The well-known difficulties of defining a phase operator of an oscillator are considered from the point of view of the canonical transformation to action and phase-angle variables. This transformation turns out to be nonbijective, i.e., it is not a one-to-one onto mapping. In order to make possible the unitarity of its representations in quantum optics we should enlarge the Hilbert space of the problem. In this enlarged space we find a phase operator that, after projection, reproduces previous candidates to represent a well-behaved phase operator in the quantum domain.
\end{abstract}

PACS number(s): 42.50.Dv, 03.65.-w

\section{INTRODUCTION}

The correct definition of phase in quantum mechanics encounters basic difficulties and has provoked a host of discussions and controversies. It seems that there is no simple and satisfactory translation of the Poisson bracket $\{j, \phi\}=1$, where $j$ and $\phi$ are the action and phase-angle variables for a harmonic oscillator, into an equivalent commutation relation in quantum mechanics $[n, \phi]=i$ in terms of the corresponding number and phase operators [1].

There are essentially two basic sources for these difficulties: the periodicity of the phase and the existence of a lower bound to the energy. However, given the relevance of such variables there have been many attempts to solve the problem, and certainly very interesting progress has been done in the past years [2].

Perhaps one of the best known solutions of the problem is that of Susskind and Glogower [3], that using the notions introduced in the pioneering work of Dirac [4], constructed the cosine and sine operators of the phase, which have been extensively used by many years, since they give reasonable physical results, at least in the classical limit.

More recently, Newton [5] overcame some of the difficulties due to the semibounded nature of the number operator by doubling the Hilbert space and formally identifying $n$ with the angular momentum component $L_{z}$ restricted to the subspace wherein $L_{z} \geq 0$. However, he dismissed the existence of a phase operator due to the periodicity of the wave functions in the phase-angle variable.

Garrison and Wong [6], and later Galindo [7], introduced a self-adjoint phase operator canonically conjugated to the number operator on some dense set of the Hilbert space. However, this operator has not attracted much attention in the quantum opticians community due to its rather complex structure that made it impractical in many senses.

Recently, the attempts of Pegg and Barnett [8] to construct a Hermitian phase operator have excited consider- able interest. Their approach starts from the existence of states of well-defined phase. They ascribe the obstacles to defining a satisfactory phase operator to the use of an infinite Hilbert space from the start, and propose to work with a finite but arbitrarily large Hilbert space of $s+1$ dimensions. With the aid of the phase states, they are able to define a Hermitian phase operator. Then, after measurable quantities are calculated, the limit $s \rightarrow \infty$ is taken.

The predictions of the Pegg-Barnett formalism for phase fluctuations have been compared with the experimental measurements of the phase fluctuations in a monomode laser beam, finding good agreement [9-11].

After the success of this approach, two interesting and sound papers due to Bergou and Englert [12] and Gantsog, Miranowicz, and Tanaś [13] have raised the question of comparing its predictions with those of Garrison and Wong. Both approaches give the same results for semiclassical states, but there are essential differences for states with few photons.

On the other hand, from the classical point of view, the question of the phase is nothing but a canonical transformation from position and momentum to action and phase-angle variables. Moshinsky and Seligman [14] have addressed this question in great detail, showing that this transformation is nonlinear and in fact nonbijective, i.e., it is not a one-to-one onto mapping. To recover the bijectivity they arrived at the need to introduce an additional variable called ambiguity spin.

In some previous works [15-17], we have analyzed the representations of these nonbijective canonical transformations in quantum optics. To get a unitary representation, it is necessary to add to the Hilbert space a new space to completely describe the system.

The aim of the present paper is just to show how the ambiguity spin can be used to unitarize the transformation and then to find a possible description of the phase in the quantum world.

\section{PHASE OPERATORS}

In his original work on the quantized electromagnetic field, Dirac [4] proposed the existence of an exponential of 
phase defined via a polar decomposition of the amplitude of the one-mode photon field in the form

$$
a=E \sqrt{n},
$$

where $n$ is the number operator. The operator $E$, nowadays known as the Susskind-Glogower phase operator, which may be written as

$$
E=\frac{1}{\sqrt{n+1}} a
$$

is unfortunately not unitary, since

$$
\begin{aligned}
& E E^{\dagger}=1, \\
& E^{\dagger} E=1-P_{0},
\end{aligned}
$$

where $P_{0}=|0\rangle\langle 0|$ is the projection on the vacuum. The action on the number basis is just as a ladder operator

$$
\begin{aligned}
E|n\rangle & =\left(1-\delta_{n, 0}\right)|n-1\rangle, \\
E^{\dagger}|n\rangle & =|n+1\rangle .
\end{aligned}
$$

Although it does not allow the existence of a truly phase operator, their non-normalized eigenstates

$$
|\phi\rangle=\frac{1}{\sqrt{2 \pi}} \sum_{n=0}^{\infty} e^{i n \phi}|n\rangle
$$

are usually considered as states of well-defined phase [18]. Such states are not orthogonal, but they allow for the resolution of the identity and for an expression of $E$ as

$$
E=\int_{\phi_{0}}^{\phi_{0}+2 \pi} d \phi|\phi\rangle e^{i \phi}\langle\phi|
$$

where $\phi_{0}$ is some arbitrary reference phase.

Newton [5] has overcome some of the difficulties due to the one-sided nature of the spectrum of the number operator by a very ingenious doubling of the Hilbert space. Let us denote by $\overline{\mathcal{H}}$ this doubled space obtained by introducing a spinlike variable with two values. We define an orthonormal basis on $\overline{\mathcal{H}}$ as

$$
|\bar{n}\rangle=\left\{\begin{array}{ll}
\left(\begin{array}{c}
|n\rangle \\
0
\end{array}\right), & n \geq 0 \\
0 \\
|-n-1\rangle
\end{array}\right), \quad n<0
$$

and now $-\infty<n<\infty$.

The operator $E$ can be extended to $\overline{\mathcal{H}}$ as

$$
\bar{E}=\left(\begin{array}{cc}
E & 0 \\
P_{0} & E^{\dagger}
\end{array}\right),
$$

and one easily finds that

$$
\begin{gathered}
\bar{E}|\bar{n}\rangle=|\bar{n}-1\rangle, \\
\bar{E}^{\dagger}|\bar{n}\rangle=|\bar{n}+1\rangle,
\end{gathered}
$$

for all integers $-\infty<\bar{n}<\infty$, and then it is a truly unitary operator. The eigenstates of $\bar{E}$, which we shall denote by $|\bar{\phi}\rangle$, are now

$$
|\bar{\phi}\rangle=\frac{1}{\sqrt{2 \pi}} \sum_{\bar{n}=-\infty}^{\infty} e^{i \bar{n} \bar{\phi}}|\bar{n}\rangle,
$$

and form an orthonormal basis on $\overline{\mathcal{H}}$.

The number operator in $\overline{\mathcal{H}}$

$$
\bar{n}=\left(\begin{array}{cc}
n & 0 \\
0 & -(n+1)
\end{array}\right)
$$

is formally a self-adjoint operator verifying the right commutation relation

$$
[\bar{E}, \bar{n}]=\bar{E} .
$$

In the $\bar{\phi}$ representation, generated by the eigenstates of $\bar{E}$, their action becomes

$$
\begin{aligned}
& \bar{E}=e^{i \bar{\phi}}, \\
& \bar{n}=i \frac{\partial}{\partial \bar{\phi}}
\end{aligned}
$$

so the operators $\bar{E}$ and $\bar{n}$ may be considered as canonically conjugate in an extended sense. The method of Newton is therefore tantamount to identifying the number operator with the angular momentum component $L_{z}$. Finally, in order to recover physical results, we must project on $\mathcal{H}$; that is, $L_{z} \geq 0$. Note that states containing a negative number of photons are inaccessible to any physical measurement, so their existence in this doubled space does not predict any new phenomena.

If we define a phase operator in $\overline{\mathcal{H}}$ as

$$
\bar{\Phi}=\int_{\bar{\phi}_{0}}^{\bar{\phi}_{0}+2 \pi} d \bar{\phi}|\bar{\phi}\rangle \bar{\phi}\langle\bar{\phi}|,
$$

then either it has to be made discontinuous at $\bar{\phi}=$ $\bar{\phi}_{0}+2 m \pi$ or else, as $\bar{\phi} f(\bar{\phi})$ is not periodic if $f(\bar{\phi})$ is, the operator is ill defined. So, Newton dismisses the existence of the phase operator as being precluded by the periodicity of the wave functions in the phase-angle variable.

However, in spite of these difficulties, let us consider

$$
\frac{1}{i}[\bar{n},|\bar{\phi}\rangle\langle\bar{\phi}|]=-\frac{\partial}{\partial \bar{\phi}}(|\bar{\phi}\rangle\langle\bar{\phi}|)
$$

to find, in a formal sense, that

$$
\frac{1}{i}[\bar{n}, \bar{\Phi}]=1-\left|\bar{\phi}_{0}\right\rangle\left\langle\bar{\phi}_{0}\right|
$$

So, on the dense set of states $|\bar{\psi}\rangle$, characterized by $\left\langle\bar{\phi}_{0} \mid \bar{\psi}\right\rangle=0$, the second term on the right-hand side vanishes, giving

$$
[\bar{n}, \bar{\Phi}]=i
$$


Thus, in this dense set, a bounded self-adjoint phase operator canonically conjugate to $\bar{n}$ does exist, which is another confirmation that the representation of canonical commutation relations is certainly a touchy business.

Since the states $|\bar{\phi}\rangle$ are orthonormal, we have

$$
\bar{\Phi}^{k}=\int_{\bar{\phi}_{0}}^{\bar{\phi}_{0}+2 \pi} d \bar{\phi}|\bar{\phi}\rangle \bar{\phi}^{k}\langle\bar{\phi}|
$$

and the unitary operator $\bar{E}$ can be expressed as

$$
\bar{E}=e^{i \bar{\Phi}}=\int_{\bar{\phi}_{0}}^{\bar{\phi}_{0}+2 \pi} d \bar{\phi}|\bar{\phi}\rangle e^{i \bar{\phi}}\langle\bar{\phi}| \text {. }
$$

To recover physical results we must finally project on $\mathcal{H}$. However, this must be done with some care, since if $P$ is the projector on $\mathcal{H}$, we have

$$
P \bar{\Phi}^{k} P \neq(P \bar{\Phi} P)^{k}
$$

for $k>1$. In this fashion, if we project the exponential of the phase

$$
P \bar{E} P=\int_{\phi_{0}}^{\phi_{0}+2 \pi} d \phi|\phi\rangle e^{i \phi}\langle\phi|,
$$

we get the Susskind-Glogower phase operator. However, if we rather focus of the operator $\bar{\Phi}$ itself, we obtain

$$
\Phi=P \bar{\Phi} P=\int_{\phi_{0}}^{\phi_{0}+2 \pi} d \phi|\phi\rangle \phi\langle\phi|,
$$

which is just the phase operator introduced by Garrison and Wong [6], and independently by Galindo [7], leading to the number-phase commutation

$$
[n, \Phi]=i
$$

on the dense domain of states $\Omega=\left\{|\psi\rangle:\left\langle\phi_{0} \mid \psi\right\rangle=0\right\}$. Without embarking on mathematical subtleties, two remarks seem in order. First, the operators $n$ and $\Phi$ define a Heisenberg pair, but as demonstrated by Rocca and Siruge [19], they do not define a Weyl pair in the sense that they do not verify

$$
e^{i \alpha n} e^{i \beta \Phi}=e^{i \beta \Phi} e^{i \alpha n} e^{i \alpha \beta}
$$

for any $\alpha$ and $\beta$ reals. Second, from (2.23) the uncertainty relation

$$
\Delta n \Delta \Phi \geq \frac{1}{2}
$$

follows, but it clearly holds true only on the domain $\Omega$, but not everywhere.

Unfortunately, when approximating even simple physical states in this dense set, one recognizes rather undesirable properties. As clearly pointed out by Bergou and Englert [12], when number states are approximated in $\Omega$, the better approximation is, the larger the spread of $n$, which is certainly unexpected from our physical intuition. Moreover, the standard concept that in a number state all the phases are equally probable, which feeds the expectation $\Delta \Phi=\pi / \sqrt{3}$, agrees with the calculation using the Garrison-Wong operator only for sufficiently large values of $n$, since one easily obtains

$$
(\Delta \Phi)^{2}=\frac{\pi^{2}}{3}-\sum_{k=n+1}^{\infty} \frac{1}{k^{2}}=\frac{\pi^{2}}{6}+\sum_{k=1}^{n} \frac{1}{k^{2}} .
$$

The Garrison-Wong phase operator can be expressed in terms of the Susskind-Glogower one as

$$
\Phi=\phi_{0}+\pi+\sum_{k=1}^{\infty} \frac{i}{k}\left(e^{-i k \phi_{0}} E^{k}-e^{i k \phi_{0}} E^{\dagger^{k}}\right)
$$

or, as expressed by Popov and Yarunin [20]

$$
\Phi=\phi_{0}+\pi+i\left[\ln \left(1-e^{-i \phi_{0}} E\right)-\ln \left(1-e^{i \phi_{0}} E^{\dagger}\right)\right] .
$$

Note that since the phase states (2.5) are not orthogonal, we have now that

$$
\Phi^{k} \neq \int_{\phi_{0}}^{\phi_{0}+2 \pi} d \phi|\phi\rangle \phi^{k}\langle\phi|
$$

for $k>1$, and for the expectation values of field states $|\psi\rangle$

$$
\left\langle\psi\left|\Phi^{k}\right| \psi\right\rangle \neq \int_{\phi_{0}}^{\phi_{0}+2 \pi} d \phi \phi^{k}|\langle\phi \mid \psi\rangle|^{2}
$$

which clearly means that $|\langle\phi \mid \psi\rangle|^{2}$ cannot be viewed here as a phase distribution function. To find the true Garrison-Wong phase distribution for the state $|\psi\rangle$ we should compute the quantity

$$
P_{\mathrm{GW}}(\Phi)=|\langle\Phi \mid \psi\rangle|^{2}=\left|\sum_{n=0}^{\infty} \psi_{n}\langle\Phi \mid n\rangle\right|^{2}
$$

where $|\Phi\rangle$ is the eigenstate of the Garrison-Wong operator. The function $\langle\Phi \mid n\rangle$ has a quite complex structure, but can be computed either numerically or from the recursive relations derived in Ref. [6].

The Pegg-Barnett formulation of the phase can be viewed in this context as a kind of orthogonalization of the Susskind-Glogower phase states. Their way to accomplish this program starts by considering a finitedimensional Hilbert space $\mathcal{H}_{s}$ spanned by $|n\rangle$, where now $n$ runs the non-negative integers up to an arbitrary but finite value of $s$. In this space they define a complete orthonormal set of phase states by

$$
\left|\tilde{\phi}_{m}\right\rangle=\frac{1}{\sqrt{s+1}} \sum_{n=0}^{s} e^{i n \tilde{\phi}_{m}}|n\rangle,
$$

where

$$
\tilde{\phi}_{m}=\phi_{0}+\frac{2 \pi}{s+1} m
$$


with $m=0,1, \ldots, s$; and then define a phase operator as

$$
\tilde{\Phi}_{s}=\sum_{m=0}^{s}\left|\tilde{\phi}_{m}\right\rangle \tilde{\phi}_{m}\left\langle\tilde{\phi}_{m}\right| \text {. }
$$

Bergou and Englert [12] and Popov and Yarunin [20] claimed that this phase operator represents a twofold approximation to the Garrison-Wong operator, inasmuch as the integral in (2.22) is replaced by a sum and the Susskind-Glogower phase states are projected on the subspace $\mathcal{H}_{s}$. However, the Pegg-Barnett prescription is to compute the expectation value of any observable of interest in $\mathcal{H}_{s}$ and, only after that, take the limit $s \rightarrow \infty$.

Note that in $\mathcal{H}_{s}$, the phase states $\left|\tilde{\phi}_{m}\right\rangle$ are orthonormal, so we can write

$$
\tilde{\Phi}_{s}^{k}=\sum_{m=0}^{s}\left|\tilde{\phi}_{m}\right\rangle \tilde{\phi}_{m}^{k}\left\langle\tilde{\phi}_{m}\right|
$$

and the expectation values in $\mathcal{H}_{s}$ can be expressed as

$$
\left\langle\psi\left|\tilde{\Phi}_{s}^{k}\right| \psi\right\rangle=\sum_{m=0}^{s} \tilde{\phi}_{m}^{k}\left|\left\langle\tilde{\phi}_{m} \mid \psi\right\rangle\right|^{2},
$$

and so the quantity $\left|\left\langle\tilde{\phi}_{m} \mid \psi\right\rangle\right|^{2}$ gives a true probability of being found in the phase state $\left|\tilde{\phi}_{m}\right\rangle$. However, when "physical states" [8] are considered, the sum may be replaced in computational problems by

$$
\lim _{s \rightarrow \infty}\left\langle\psi\left|\tilde{\Phi}_{s}^{k}\right| \psi\right\rangle=\int_{\phi_{0}}^{\phi_{0}+2 \pi} d \phi \phi^{k} P_{\mathrm{PB}}(\phi),
$$

where the continuous phase distribution $P_{\mathrm{PB}}(\phi)$ is defined by

$$
P_{\mathrm{PB}}(\phi)=\lim _{s \rightarrow \infty} \frac{s+1}{2 \pi}\left|\left\langle\tilde{\phi}_{m} \mid \psi\right\rangle\right|^{2}=|\langle\phi \mid \psi\rangle|^{2}
$$

and $\tilde{\phi}_{m}$ has been replaced by the continuous phase variable $\phi$. Therefore, the $P_{\mathrm{PB}}$ function is obtained by projecting the state on the corresponding Susskind-Glogower phase states and this formalism can be derived from the Newton approach since

$$
\lim _{s \rightarrow \infty}\left\langle\psi\left|\tilde{\Phi}_{s}^{k}\right| \psi\right\rangle=\left\langle\psi\left|P \bar{\Phi}^{k} P\right| \psi\right\rangle=\left\langle\psi\left|\bar{\Phi}^{k}\right| \psi\right\rangle
$$

Thus, in the limit $s \rightarrow \infty$ the Pegg-Barnett approaches the Garrison-Wong operator [21], although this limit is a delicate matter, since

$$
\lim _{s \rightarrow \infty} \tilde{\Phi}_{s}=\Phi
$$

but

$$
\lim _{s \rightarrow \infty} \tilde{\Phi}_{s}^{2} \neq \Phi^{2}
$$

which illustrates the fact that, in general

$$
\lim _{s \rightarrow \infty} f\left(\tilde{\Phi}_{s}\right) \neq f\left(\lim _{s \rightarrow \infty} \tilde{\Phi}_{s}\right)=f(\Phi)
$$

which can be viewed as a consequence of (2.20).
Then, although the Pegg-Barnett and Garrison-Wong operators may appear superficially to approximate each other, there are very important differences in the two approaches which lead to quite different predictions, because the results obtained depend critically on the order in which this limit is taken.

Recently Gantsog, Miranowicz, and Tanaś [13] have compared in detail the predictions of the GarrisonWong with the Pegg-Barnett approach. Their conclusion is that the Garrison-Wong formalism introduces some anisotropy in the phase distribution function which seems to be inconsistent with the symmetry showed by the Wigner and $Q$ functions of the corresponding field states. The Pegg-Barnett formalism does not suffer from this "symmetry-breaking" problem. So, really there is a qualitative difference between the two formalisms in predicting phase properties of the fields, although in the limit of high excitation numbers all the phase operators discussed here give the same predictions.

\section{QUANTUM ACTION AND PHASE-ANGLE VARIABLES FOR A HARMONIC OSCILLATOR}

A canonical transformation is often interpreted as providing a new set of coordinates in phase space which are on an equal footing with the original ones. It seems also generally accepted that canonical transformations are represented in quantum mechanics by unitary operators.

Thus it seems reasonable, at least in principle, to consider the transformation passing from the Cartesian coordinates position and momentum $(q, p)$ in the phase plane to the action and phase-angle variables corresponding to an oscillator of unit mass and frequency, i.e.,

$$
\begin{gathered}
j=\frac{1}{2}\left(p^{2}+q^{2}\right), \\
\phi=\arctan \left(\frac{p}{q}\right) .
\end{gathered}
$$

Next, we could ask for a unitary representation in quantum mechanics, translating the classical relations (3.1) between $c$ numbers into relations between operators, which can be defined in the form

$$
\begin{aligned}
& U q U^{\dagger}=n, \\
& U p U^{\dagger}=\phi,
\end{aligned}
$$

in units $\hbar=1$. Note that the quantum analog of the classical action is now the number operator. The explicit knowledge of this representation will provide us with a phase-angle operator canonically conjugated to the number operator.

It is not necessary to go too far on this approach to run into serious troubles. It can be expected that the difficulties in the definition of a well-behaved phase operator are intimately linked to the problem of finding the 
unitary representation for the nonlinear transformation to action and phase-angle variables [14]. So our purpose in this section is to relate these two problems and show how the approaches discussed in Sec. II appear in this context. We are going to see that the requirement of a unitary representation leads to the phase operators of the preceding section, also showing the way in which they are related.

The obstacles to the unitarity of $U$ arise from the different spectra of the operators to be related and the periodic character of the phase, making the transformation not only a nonlinear but also a nonbijective one. Focusing on (3.2a), we can see that while the spectrum of $q$ runs continuously from $-\infty$ to $\infty$, the one of the number operator is not only discrete, but also bounded from below.

If we denote by $|q\rangle$ the eigenstates of $q$, we can always write the eigenvalues in the form

$$
q=\bar{n}+\lambda,
$$

where $0 \leq \lambda<1$ and $\bar{n}$ is an integer. The spectra of $q$ and $n$ can then be identified when we restrict ourselves to a fixed $\lambda$ and to the non-negative values of $\bar{n}$.

Following Dirac's program [22], a family of operators closer to what we are looking for could be given by $[23,24]$

$$
U_{\lambda}=\sum_{n=0}^{\infty}|n\rangle\langle q=n+\lambda|,
$$

which clearly is not unitary.

If we require a unitary transformation, the program outlined by Moshinsky and Seligman goes through the enlargement of the image space to provide a variable, called the ambiguity spin, which can be used to equalize the spectra. This enlargement must be done here in two ways. In first place, we must include eigenstates of the number operator with negative integer eigenvalues to solve the problem caused by the semiboundedness of the number operator, in much the same way as proposed by Newton. In second place, it is necessary to add a continuous variable in the interval $[0,1)$ as it is the $\lambda$ variable in (3.3).

Then, we can consider as image space the product $\overline{\mathcal{H}} \otimes \Lambda$, where $\overline{\mathcal{H}}$ is the extension of $\mathcal{H}$ including the negative number states, and $\Lambda$ is a Hilbert space needed to accommodate the new continuous variable [24]. We shall denote by $|\lambda\rangle$ an orthonormal basis in this space $\Lambda$.

In this way, a unitary operator can be given in the form $U: \mathcal{H} \longrightarrow \overline{\mathcal{H}} \otimes \Lambda$,

$$
U=\int_{0}^{1} d \lambda \sum_{\bar{n}=-\infty}^{+\infty}|\bar{n}\rangle|\lambda\rangle\langle q=\bar{n}+\lambda| .
$$

The final form for $U q U^{\dagger}$ is

$$
U q U^{\dagger}=\sum_{\bar{n}=-\infty}^{+\infty} \bar{n}|\bar{n}\rangle\left\langle\bar{n}\left|+\int_{0}^{1} d \lambda\right| \lambda\right\rangle \lambda\langle\lambda|
$$

We note that the image of $q$ gives separate contributions over the $\overline{\mathcal{H}}$ and $\Lambda$ spaces, and that the first term can be identified as the third component of an angular momentum.

To obtain $U$ we have used only the relation (3.2a) defining the transformation. The reason for not using (3.2b) is that we do not want to make any assumption about the form of $\phi$. As a consequence of this procedure, $U$ can not be completely determinated. It still could be added in (3.5) a relative phase depending on $\bar{n}$ and $\lambda$. However, the main features of this procedure are independent of this choice, and we shall use this form for $U$.

The problems associated with the periodicity can be avoided working with periodic functions of the phase. The result for the exponential of the phase is

$$
U e^{i p} U^{\dagger}=\sum_{\bar{n}=-\infty}^{+\infty}|\bar{n}-1\rangle\langle\bar{n}| .
$$

It can be recognized that this is precisely the exponential of the phase operator, acting on the extended space $\overline{\mathcal{H}}$, introduced by Newton (2.9). Then, all his phase formalism can be obtained in this context from (3.7). Note that in this case we have not started from any assumption about the phase operator. Moreover, as we showed in Sec. II, the Susskind-Glogower, Garrison-Wong, and Pegg-Barnett phase operators can be derived from (3.7) with the appropriate interpretation of the final projection over the space $\mathcal{H}$.

The loss of the good properties of the phase operator (3.7) after the projection can be understood here in connection with corresponding loss of unitarity of the transformation.

Next we briefly introduce a basis in the original Hilbert space that illustrates the nonbijectivity of the transformation and the introduction of the ambiguity spin.

As far as the transformation identifies $p$ with the phase, which must have a periodic character that $p$ does not have (making the transformation nonbijective), it seems natural to classify the states in the original space $\mathcal{H}$ according to their properties under translations by $2 \pi m$ of $p$. This is precisely the ambiguity group introduced by Moshinsky and Seligman.

In consequence, it is advantageous to work with a basis of eigenvectors of the translation operator

$$
T_{p}=e^{i 2 \pi q} \text {. }
$$

Since this operator does not form by itself a complete set, it is necessary to add another one, a suitable and natural choice being

$$
T_{q}=e^{i p}
$$

These two operators form a complete set of commuting operators [25] and we denote the associated basis by $|\phi, \lambda\rangle$, in such a way that

$$
\begin{aligned}
& T_{p}|\phi, \lambda\rangle=e^{i 2 \pi \lambda}|\phi, \lambda\rangle, \\
& T_{q}|\phi, \lambda\rangle=e^{i \phi}|\phi, \lambda\rangle,
\end{aligned}
$$

where $\phi \epsilon[0,2 \pi)$ and $\lambda \epsilon[0,1)$. Denoting by $|p\rangle$ an eigen- 
state of the momentum operator $p$, we have

$$
|\phi, \lambda\rangle=e^{-i \lambda \phi} \sum_{k=-\infty}^{+\infty} e^{-i 2 \pi \lambda k}|p=2 \pi k+\phi\rangle .
$$

The main advantage of this basis lies in the introduction of an anglelike variable $\phi$ related with $p$ that could be interpreted, in view of (3.11), as its periodic part, and that can be useful dealing with the periodicity problem. In fact, any $2 \pi$ periodic function of $p$ is only function of $\phi$. We could also define a $\Phi_{p}$ operator as

$$
\Phi_{p}=\int_{0}^{2 \pi} d \phi \int_{0}^{1} d \lambda|\phi, \lambda\rangle \phi\langle\phi, \lambda|,
$$

and we have

$$
e^{i p}=\int_{0}^{2 \pi} d \phi \int_{0}^{1} d \lambda|\phi, \lambda\rangle e^{i \phi}\langle\phi, \lambda|=e^{i \Phi_{p}} .
$$

So, in the $|\phi, \lambda\rangle$ representation, any $2 \pi$ periodic function $f(p)$ acts multiplicatively as $f(\phi)$. On the other hand, in the same representation, $q$ acts on the form $q \rightarrow i \partial_{\phi}+\lambda$. We can see that the first term formally coincides again with the third component of an angular momentum.

Thus, we are able to extract from the original space a phase-angle variable and its conjugate one. Moreover, the variable $\lambda$ is essential to completely describe the physical space, which reflects the fact that $p$ is not periodic. Then, to fulfill (3.2) with a unitary operator we need to enlarge the image space to accommodate the $\lambda$ variable. This extension solves both the difficulties associated with the periodicity of the phase and the continuous spectrum of $q$. To solve the problems caused by the semibound- edness of the number operator we must extend also its spectrum to the negative integers as it were an angular momentum. The way to do these enlargements is the same as discussed before. Finally note that the phase operator (2.14) is the image by $U$ of the $\Phi_{p}$ operator (3.12).

\section{CONCLUSIONS}

The difficulties in the translation into quantum mechanics of the phase-angle variable have lead to the introduction of several phase operators. We have found that the same troubles arise in the representation in quantum mechanics of the nonlinear canonical transformation to action and phase angle.

We have shown that the solution proposed for its unitary representations provides a route to define a phase operator that does not need any previous assumption about its form. This formalism not only contains previous approaches to the problem, but also shows their relationships.

\section{ACKNOWLEDGMENTS}

The authors would like to thank Professor A. Galindo and Professor R. Tanaś for a critical reading of the manuscript and useful comments. They are grateful as well to Professor J. F. Cariñena for helpful and enlightening discussions of some rather technical points. Finally, they benefited from the continuous interest and advice of Professor E. Bernabeu.
[1] P. Carruthers and M. M. Nieto, Rev. Mod. Phys. 40, 441 (1968).

[2] S. M. Barnett and D. T. Pegg, J. Phys. A 19, 3849 (1986).

[3] L. Susskind and J. Glogower, Physics 1, 49 (1964).

[4] P. A. M. Dirac, Proc. R. Soc. London Ser. A 114, 243 (1927).

[5] R. G. Newton, Ann. Phys. (N.Y.) 124, 327 (1980).

[6] J. C. Garrison and J. Wong, J. Math. Phys. 11, 2242 (1970).

[7] A. Galindo, Lett. Math. Phys. 8, 495 (1984); 9, 263 (1985).

[8] D. T. Pegg and S. M. Barnett, Europhys. Lett. 6, 483 (1988); J. Mod. Opt. 36, 7 (1989).

[9] R. Lynch, Phys. Rev. A 41, 2841 (1990).

[10] C. C. Gerry and K.E. Urbańsky, Phys. Rev. A 42, 662 (1990).

[11] A. Bandilla, Opt. Commun. 80, 267 (1991).

[12] J. Bergou and B. G. Englert, Ann. Phys. (N.Y.) 209, 479 (1991).

[13] T. Gantsog, A. Miranowicz, and R. Tanaś, Phys. Rev. A 46, 2870 (1992).
[14] M. Moshinsky and T. H. Seligman, Ann. Phys. (N.Y.) 114, 243 (1978); J. Phys. A 12, L135 (1979).

[15] A. Luis and L. L. Sánchez-Soto, J. Phys. A 24, 2083 (1991).

[16] A. Luis and L.L. Sánchez-Soto, Phys. Rev. A 47, 1492 (1993).

[17] A. Luis and L.L. Sánchez-Soto, Quantum Opt. 5, 33 (1993).

[18] J. M. Lévy-Leblond, Ann. Phys. (N.Y.) 101, 319 (1976).

[19] F. Rocca and M. Siruge, Commun. Math. Phys. 34, 111 (1973).

[20] V. N. Popov and V. S. Yarunin, Vestn. Leningr. Univ. N22, 7 (1973); J. Mod. Opt. 39, 1525 (1992).

[21] S. M. Barnett and D. T. Pegg, J. Mod. Opt. 39, 2121 (1992).

[22] P. A. M. Dirac, Quantum Mechanics (Clarendon, Oxford, 1958).

[23] B. Leaf, J. Math. Phys. 10, 1980 (1969).

[24] J. F. Plebański and T. H. Seligman, Rep. Math. Phys. 17, 437 (1980).

[25] J. Zak, Phys. Rev. 168, 686 (1968). 